\title{
Fuse Device
}

National Cancer Institute

\section{Source}

National Cancer Institute. Fuse Device. NCI Thesaurus. Code C49966.

An electrical device designed to stop the flow of current when an overload condition exists. 